\title{
Loss and retention of resistance genes in five species of the Brassicaceae family
}

\author{
Hanneke M Peele", Na Guan, Johan Fogelqvist and Christina Dixelius
}

\begin{abstract}
Background: Plants have evolved disease resistance (R) genes encoding for nucleotide-binding site (NB) and leucine-rich repeat (LRR) proteins with N-terminals represented by either Toll/Interleukin-1 receptor (TIR) or coiled-coil (CC) domains. Here, a genome-wide study of presence and diversification of CC-NB-LRR and TIR-NB-LRR encoding genes, and shorter domain combinations in 19 Arabidopsis thaliana accessions and Arabidopsis lyrata, Capsella rubella, Brassica rapa and Eutrema salsugineum are presented.

Results: Out of $528 R$ genes analyzed, 12 CC-NB-LRR and 17 TIR-NB-LRR genes were conserved among the $19 \mathrm{~A}$. thaliana genotypes, while only two CC-NB-LRRs, including ZARI, and three TIR-NB-LRRs were conserved when comparing the five species. The RESISTANCE TO LEPTOSPHAERIA MACULANS 1 (RLM1) locus confers resistance to the Brassica pathogen L. maculans the causal agent of blackleg disease and has undergone conservation and diversification events particularly in B. rapa. On the contrary, the RLM3 locus important in the immune response towards Botrytis cinerea and Alternaria spp. has recently evolved in the Arabidopsis genus.
\end{abstract}

Conclusion: Our genome-wide analysis of the $R$ gene repertoire revealed a large sequence variation in the 23 cruciferous genomes. The data provides further insights into evolutionary processes impacting this important gene family.

Keywords: Arabidopsis thaliana, Brassicaceae, CC/TIR-NB-LRR domains, Genomes, Leptosphaeria maculans, Resistance genes

\section{Background}

As sessile organisms, plants have adapted to their changing surroundings and their survival is based primarily on timely evolved immune responses. The first line of defense occurs at the plant cell surface with the recognition of conserved microbial groups such as lipopolysaccharides and peptidoglycans, commonly revered to as pathogen or microbeassociated molecular patterns (PAMPs/MAMPs). The MAMPs are recognized by cognate pattern-recognition receptors (PRRs) and trigger immediate immune responses leading to basal PAMP-triggered immunity (PTI) [1,2]. Known PRRs fall into one of two receptor classes: transmembrane receptor kinases and transmembrane receptorlike proteins, the latter of which lack any apparent internal signaling domain [3]. Notably, PRRs are components of multiprotein complexes at the plasma membrane under tight control by protein phosphatases and other regulatory

\footnotetext{
* Correspondence: hanneke.peele@slu.se Department of Plant Biology, Swedish University of Agricultural Sciences, Uppsala BioCenter, Linnean Center for Plant Biology, P.O. Box 7080, S-75007 Uppsala, Sweden
}

(c) 2014 Peele et al.; licensee BioMed Central Ltd. This is an Open Access article distributed under the terms of the Creative Commons Attribution License (http://creativecommons.org/licenses/by/4.0), which permits unrestricted use, distribution, and reproduction in any medium, provided the original work is properly credited. The Creative Commons Public Domain Dedication waiver (http://creativecommons.org/publicdomain/zero/1.0/) applies to the data made available in this article, unless otherwise stated.

proteins [4]. In a number of cases specialized pathogens are able to overcome basal PTI by either circumventing the detection of PAMPs or interfering with PTI by delaying, suppressing or reprogramming host responses via delivery of effector molecules inside host cells. As a counter mechanism, deployed intracellular resistance $(\mathrm{R})$ proteins detect the presence of these effectors directly or indirectly leading to effector-triggered immunity (ETI). The RPM1INTERACTING PROTEIN 4 (RIN4) is a well-studied key-player in the former situation [5,6], whereas direct interaction could be exemplified by the $R$ genes and effectors in the rice - Magnaporthe oryzae pathosystem [7,8].

The plant resistance proteins are modular, that is, they consist of combinations of conserved elements some with features shared with animals reviewed by [9-11]. The majority of $\mathrm{R}$ proteins are typically composed of a nucleotide-binding site $(\mathrm{NB})$ with a leucine-rich repeat (LRR) domain of variable length at the C-terminus. These NB-LRR proteins are divided into two classes on the basis of their $\mathrm{N}$-terminal sequences consisting either of a coiled-coil (CC) sequence or of a domain that 
shares sequence similarity with the Drosophila melanogaster TOLL and human interleukin-1 receptor referred to as TIR. These blocks of conserved sequences have remained throughout evolution and can still be identified in diverse organisms of eubacteria, archaea, metazoans and bryophytes [12]. Despite this high degree of conservation, the $\mathrm{R}$ proteins confer resistance to a broad spectrum of plant pathogens, including viruses, bacteria, fungi, oomycetes and nematodes [13-15].

NB-encoding resistance genes have been annotated in many monocot and dicot species pioneered by Arabidopsis thaliana [16]. The current wealth of genomes of sequenced plant species has revealed $R$ genes to be one of the largest plant gene families. In the reference genome of A. thaliana, 149 R-proteins harbor a LRR motif whereof 83 are composed of TIR-NB-LRR and 51 have CC-NB-LRR domains $[17,18]$. Several shorter proteins also are present comprising one or two domains represented by 19 TIR-NB encoding genes and 30 genes with TIR-X domains. In total, A. thaliana has approximately $\sim 200$ proteins with one to three $R$ gene-associated protein domain combinations.

In this study we took advantage of the accelerating genome information in A. thaliana and performed genomewide analyses of $R$ genes in $19 \mathrm{~A}$. thaliana genomes. We further expanded the analysis by including the genomes of the related Arabidopsis lyrata, Capsella rubella, Brassica rapa and Eutrema salsugineum species. In addition we selected two loci harboring resistance to Brassica fungal pathogens in order to trace down their evolutionary patterns. We found that $29 R$ genes formed a core set within $A$. thaliana, whereas as few as five $R$ genes were retrieved from the genomes of the five different species. One of those five genes, the HOPZ-ACTIVATED RESISTANCE 1 (ZAR1) gene known to possess novel signaling requirements is also present in other plant families within the Rosid clade. The RESISTANCE TO LEPTOSPHAERIA MACULANS 1 (RLM1) locus was partly conserved in $A$. lyrata and C. rubella and greatly diversified in B. rapa and E. salsugineum, while the RLM3 locus has recently evolved in the Arabidopsis genus. This work provides aspects on $R$ gene diversity and choice of reference genotype in comparative genomic analysis.

\section{Results}

\section{A core set of $29 R$ genes is present in $19 A$. thaliana genomes}

To gain insight on the level of $R$ gene conservation in $A$. thaliana, we analyzed the reference genome of Col- 0 and 18 additional accessions (Bur- 0 , Can- 0 , Ct- 1 , Edi- 0 , Hi- 0 , Kn-0, Ler-0, Mt-0, No-0, Oy-0, Po-0, Rsch-4, Sf-2, Tsu-0, Wil-2, Ws-0, Wu- 0 and $\mathrm{Zu}-0$ ) [19]. These 18 genomes were chosen primarily for their sequence quality, high coverage, RNA sequencing data and de novo assembly.
Pfam homology and COILS server searches on the predicted 148 NB-LRR-encoding genes [18] resulted in a reduced list of $124 R$ genes in Col-0 for further analysis, comprising 48 CC-NB-LRR (CNLs) and 76 TIR-NB-LRRs (TNLs) (Additional file 1: Table S1). Between 97 (Edi-0) to 109 (Hi-0 and Po- 0 ) of these $R$ genes were found within the genomes of the 18 newly sequenced $A$. thaliana accessions (Figure 1A, B). No additional $R$ genes besides those present in Col-0 were found in the trace sequence archives of the 18 genomes.

In a comparison of the $48 \mathrm{CNL}$ encoding genes in Col-0, between 27 (Edi- 0 ) to 40 ( $\mathrm{Hi}-0)$ were recovered in the selected accessions (Figure 1A). The protein products of the remaining genes orthologous to the CNL proteins in Col-0 were either missing one or several domains $(\mathrm{CN}, \mathrm{NL}, \mathrm{N}$ or $\mathrm{L})$ or were completely absent in at least one accession (Figure 1C). Representatives of known defense-related genes that were absent included RPS5 in Edi-0, No-0 and Sf-2, and ADR1 in Zu-0. For gene abbreviations, see Additional file 2: Table S2. In the TNL group, the number of complete TNL genes varied between 49 (No-0) and 59 (Po-0 and Wu-0) (Figure 1B, D). Examples of missing genes were RPP5 in Ct-1, Mt-0, Oy- 0 and Wu-0, and SNC1 in Can-0, Edi-0, No-0, Rsch-4, Tsu- 0 and $\mathrm{Wu}-0$.

In summary, a rather wide distribution of $R$ gene repertoires was found among the $19 \mathrm{~A}$. thaliana accessions. Out of the 124 encoding $R$ genes in Col-0, 41 genes had orthologs in the other 18 accessions. However, 12 of these genes lacked one or two domains in at least one accession. For example, RPP13 had lost its LRR domain in No-0, Rsch-4, Wil-2 and $\mathrm{Zu}-0$. In the remaining core set of $12 \mathrm{CNL}$ and $17 \mathrm{TNL}$ encoding genes, all randomly distributed over the genome (Additional file 3: Figure S1), nine genes (ADR1-L1, ADR1-L2, LOV1, RPS2, RPS4, RPS6, SUMM2, TTR1 and ZAR1), are known to be implicated in various plant defense responses.

\section{Five NB-LRR genes are conserved in five members of the Brassicaceae family}

To expand the analysis on $R$ genes in A. thaliana, we monitored possible conservation of $R$ genes across lineages in Brassicaceae represented by $A$. lyrata, C. rubella, B. rapa and E. salsugineum. Pfam homology and COILS server searches identified 404 proteins with CNL or TNL architecture (Additional file 1: Table S1). The number of predicted CNL and TNL encoding genes varied greatly: E. salsugineum (67), C. rubella (75), A. thaliana Col-0 (124), A. lyrata (127), and $B$. rapa (135), numbers that do not reflect the genome sizes or number of predicted gene models in the individual species.

Orthologous sequences in the five species were identified by phylogenetic analysis of the NB domains in the 


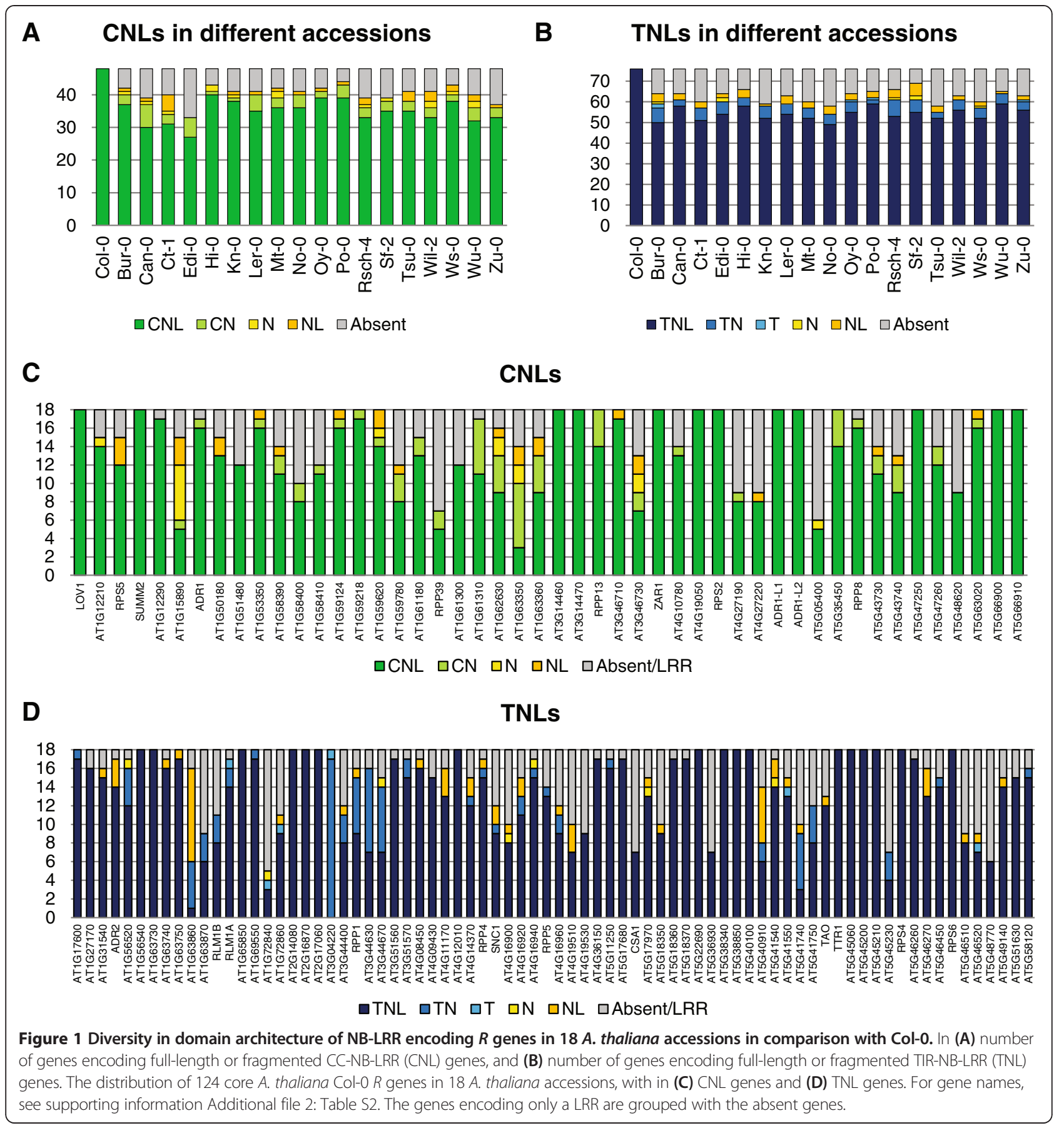

CNL and TNL sequences. In the resulting phylogenetic tree, 57 clades with orthologs from at least two plant species were formed (Additional file 4: Figure S2 and Additional file 5: Table S3). Within these 57 clades, multicopy genes from single species were also found identified as in-paralogous sequences within that specific species. The placement of the sequences outside the 57 clades was not resolved. Within the orthologous sequences a bias towards the TNL group was seen, with 52 out of 76 A. thaliana TNL sequences having an ortholog in one or more species, while only 17 out of 48 CNLs had an ortholog. Excluding in-paralogous genes, the highest number of orthologous sequences was identified between $A$. thaliana and A. lyrata (Figure 2), as concurrent with earlier findings $[20,21]$. From the $A$. thaliana core set of 29 genes, 7 CNL and 9 TNL genes were also found within two or more species including ADR1-L1, ADR1-L2, RPS2, RPS6, TTR1 and ZAR1.

In total, two CNL clades and three TNL clades with sequences from all five species were identified. Only one 

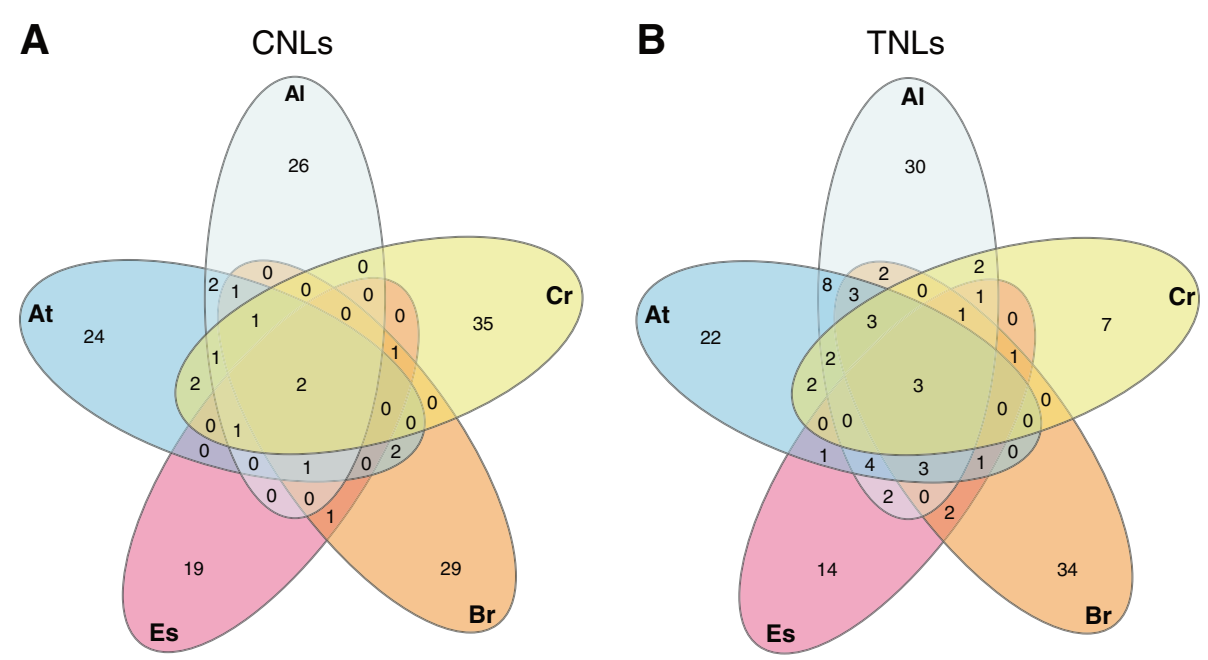

Figure $2 \mathrm{R}$ gene orthology between A. thaliana, A. lyrata, C. rubella, B. rapa and E. salsugineum. In (A) the CNL orthologs and in (B) orthologous TNL sequences in A. thaliana Col-0 (At), A. lyrata (A), C. rubella $(C r), B$. rapa (Br) and E. salsugineum (Es). Data derived from the phylogenetic analysis (Additional file 4: Figure S2).

of these clades (no. 5; Additional file 4: Figure S2) contained a gene implicated in defense responses, known as $Z A R 1$ and required for recognition of the Pseudomonas syringae T3SE HopZ1a effector [22]. ZAR1 has homologs in several species within the Rosid clade as well as in Vitis vinifera and Solanum species, and in our dataset ZAR1 was well conserved, with a $\mathrm{Ka} / \mathrm{Ks}$ ratio of 0.4 supporting purifying selection. Two other genes, At5g66900 and At5g66910 were found in the same clade (no. 12; Additional file 4: Figure S2), suggesting that they were paralogous to each other and possibly have redundant functions. In this clade, B. rapa and E. salsugineum were represented with three and two genes, respectively, while there was a single gene from A. lyrata and C. rubella. Phylogenetic analysis of the CDS sequences revealed that only the At5g66900 gene was conserved among the five species (Additional file 6: Figure S3). The RPS2 gene was earlier found in several Brassica species, including $B$. montana, B. rapa and B. oleracea [23,24], and it has most likely a homolog (945467, identity of $94 \%$ ) in $A$. lyrata [20]. In our dataset, the A. thaliana RPS2 gene was also identified in E. salsugineum but not in C. rubella. However, a BLASTN homology search, revealed similarity between RPS2 and a region annotated on the anti-sense strand as a gene without any domains in $C$. rubella (Carubv10005994m). The high similarity and identity of 88.7 suggested a possible third CNL gene being conserved among the five species.

In summary, orthology with two CNL genes (At3g50950 and At5g66900) with the possible addition of RPS2 and three TNL genes (At4g19510, At5g45230, At5g17680) was observed in all five species. Within the 19 genomes of $A$. thaliana only the CNL genes were conserved in this particular genomic comparison. No known function has been attributed to four out of the five conserved genes, including their orthologs.

\section{Conservation and diversification of the RLM1 locus}

L. maculans is a hemitrophic fungal pathogen and the causal agent of the widespread blackleg disease of Brassica crops [25]. The RLM1 locus in A. thaliana Col-0 was earlier identified as displaying important roles in the immune response [26] and contains seven genes with TNL architectures spanning between At1g63710 and At1g64360 (Additional file 7: Figure S4). Two genes, RLM1A and $R L M 1 B$ were found to be responsible for RLM1 activity, with $R L M 1 A$ as the main player in the immune response [26]. No function is known for the remaining five RLM1C-RLM1G genes. Diversification in resistant loci in different accessions has been demonstrated in several cases $[21,27,28]$ and to expand our knowledge on $R L M 1$, we studied the presence and diversification of RLM1 in our genomic data set.

Here, we found RLM1A to be present in all $18 \mathrm{~A}$. thaliana accessions encoding all three domains in fourteen accessions (Can-0, Ct- 1 , Edi- 0 , Hi-0, Ler- 0 , Mt-0, No-0, Po-0, Sf-2, Tsu-0, Wil-2, Ws-0, Wu-0 and Zu-0 (Additional file 8: Table S4). This is in agreement with their resistance phenotype [29]. In general the RLM1A genes in 17 accessions had very few variable sites compared to RLM1A in Col-0 ( $p$-distance 0.2 to $0.9 \%)$. Ws- 0 was atypical and diverged most with 230 variable sites in comparison to RLM1A in Col-0 resulting in a $p$-distance of $13.8 \%$ (Figure $3 \mathrm{~A}$ and Additional file 9: Table S5). No RLM1A homologs were identified in the $A$. lyrata, B. rapa and E. salsugineum genomes. One $R L M 1 A$ candidate was found un-annotated in the C. rubella genomic sequence and RNA expression data of the LRR region [30] suggests that this gene is expressed, and 


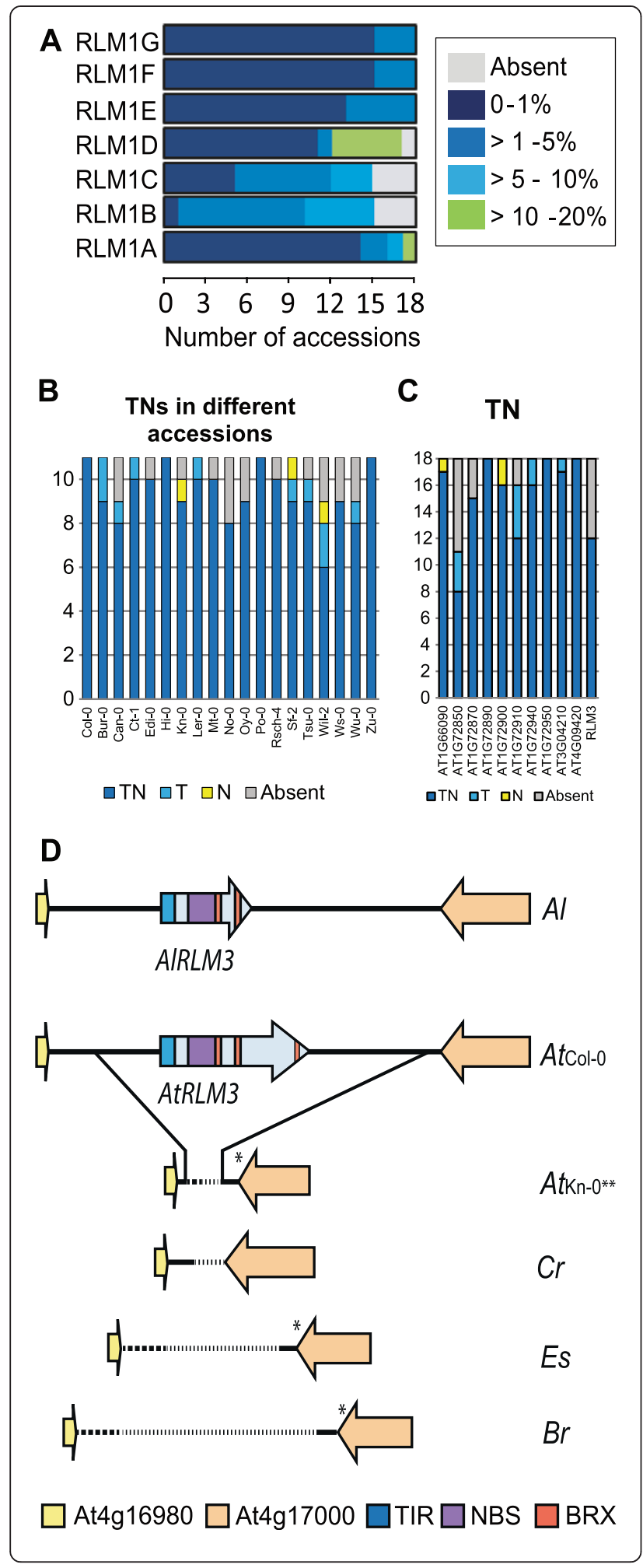

Figure 3 The TNL genes within the RLM1 locus, TN genes in 19 A. thaliana accessions and the RLM3 locus. In (A) p-distance of the different TNL encoding proteins in the RLM1 locus in the $19 \mathrm{~A}$. thaliana accessions. Details on individual gene values see supporting information Additional file 9: Table S5. Domain architecture diversity of TIR-NB encoding $R$ genes in $18 \mathrm{~A}$. thaliana accessions in comparison with Col-0 with (B) total full-length or fragmented TIR-NB (TN) genes, and (C) distribution of 11 Col-0 TN proteins in 18 A. thaliana accessions. The genes encoding only a LRR are grouped with the absent genes. (D) Synteny in the RLM3 locus between A. thaliana Col-0, A. thaliana Kn-0, A. Iyrata (A), C. rubella (Cr), B. rapa (Br) and E. salsugineum (Es). *Early stop codon; **RLM3 locus in Rsch-4, Tsu-0, Wil-2, Ws-0 and Wu-0 are identical to $\mathrm{Kn}-0$.

might have a potential role in defense responses. To support our findings, PCR amplification and sequencing of the RLM1A region in A. lyrata, B. rapa and C. rubella confirmed that only C. rubella has maintained $R L M 1 A$. B. rapa species are not known to host resistance to $L$. maculans [31] except the weedy relative $B$. rapa ssp. sylvestris $[32,33]$. In order to clarify the presence of $R L M 1 A$ we used $R L M 1 A$ specific primers to amplify this region in $B$. napus cv. Surpass 400 harboring resistance traits from the wild $B$. rapa relative, the gene progenitor, and for comparison, a known susceptible $B$. rapa genotype. Here, only $B$. rapa ssp. sylvestris contained a genomic sequence highly similar to the RLM1A gene of $A$. thaliana (identity $81 \%$ ).

The $R L M 1 B$ gene has a minor role in the immune response and is flanked by RLM1C and RLM1D. These three TNL genes encoded proteins lacking one or more domains in most of the 18 accessions in comparison to Col-0, especially RLM1D (Additional file 8: Table S4). One possible candidate orthologous to $R L M 1 C$ was found in the genomic sequence of $C$. rubella but using the annotation of $A$. thaliana for comparison the potential gene had multiple stop codons. Similarity was found for the $R L M 1 B$ to RLM1C genes in the genome of A. lyrata, B. rapa and E. salsugineum (Additional file 7: Figure S4). Due to the lack of orthology between species this chromosomal region seems to be under positive selection, showing a reduction of the RLM1B to RLMD genes within A. lyrata and $E$. salsugineum. In $B$. rapa on the contrary an expansion was observed with five TNL and one TN genes annotated to the RLM1B-RLM1D region, showing similarity to the RLM1B and RLM1C genes of A. thaliana Col-0.

The most conserved sequence within the $A$. thaliana accessions were $R L M 1 E, F$ and $G$ genes which displayed only a few modifications ( $p$-distance $0.5-0.8 \%$ ) (Additional file 9: Table S5). Further conservation was observed for RLM1F and RLM1G in A. lyrata, the latter containing two orthologs to the RLM1F and RLM1G genes with $\mathrm{Ka} / \mathrm{Ks}$ ratios of 1.3 and 0.8 in comparison to A. thaliana Col-0. Additionally, similarity was found for $R L M 1 G$ to the genomic region in C. rubella ( $\mathrm{Ka} / \mathrm{Ks}$ ratio of 0.7$)$ and transcript data has previously revealed that RLM1G is 
expressed in C. rubella [30]. In B. rapa, five TNL encoding genes were found to be orthologous to $R L M 1 F$ and RLM1G (clade no. 21, Additional file 4: Figure S2), but only two were found in the RLM1 locus. The three other TNL encoding genes were located elsewhere with no synteny with the RLM1 locus. No orthology was found for the RLM1E to RLM1G genes in E. salsugineum.

Overall, in the A. thaliana accessions the RLM1 locus is conserved in the RLM1E to RLM1G region and appears to have experienced diversification in the RLM1A to RLM1D sequence stretch. An exception was $\mathrm{Wu}-0$, in which the RLM1 locus was highly similar to the RLM1 locus in Col0 , with only an average $p$-distance of $0.2 \%$ (Additional file 9: Table S5). In the other four species, several of the RLM1 genes have experienced diversification in comparison to $A$. thaliana as well as to each other. The exception is the conserved RLM1G in both A. lyrata and C. rubella and the RLM1F in A. lyrata while RLM1A was also found in C. rubella.

\section{The RLM3 locus is unique for $A$. thaliana and A. lyrata} The RLM3 gene is of importance for immune responses not only to L. maculans but also to Botrytis cinerea and Alternaria species [34]. The gene encodes TIR and NB domains, but lacks a LRR domain. Instead, the C-terminal end contains three copies of the DZC (disease resistance, zinc finger, chromosome condensation) or BRX domain (brevis radix) originally described having a role in root development [35]. In addition to RLM3, 18 genes in A. thaliana Col-0 contain TN genes without LRR domains [18]. However, RLM3 is the only TN gene in the A. thaliana reference genome that contains BRX domains. To gain more insight on the TN encoding genes in $A$. thaliana Col-0, a Pfam homology and COILS server search was employed. This was designed to exclude genes with truncated TIR or NB domain, resulting in eleven TN genes (Additional file 1: Table S1). The presence of the TN encoding genes was further investigated in the 18 additional A. thaliana genomes.

Overall, we found between six (Wil-2) and eleven (Hi-0, Po- 0 and $\mathrm{Zu}-0$ ) genes encoding both the entire TIR and NB domain (Figure 3B). Of the eleven TN genes in Col-0, seven were present in all 18 accessions, with three encoding the complete TN. The remaining four genes encoded modifications $(\mathrm{T}$ or $\mathrm{N})$ in at least one accession (Figure $3 \mathrm{C}$ ). At1g72850 was absent in most accessions (Can-0, Edi-0, Mt- 0 , No- 0 , Oy- 0 , Wil- 2 and Ws- 0 ) and encoding only a TIR domain in Bur-0, Ct-1 and Sf-2. When we expanded the Pfam homology searches we found seven TNs in $A$. lyrata, one in C. rubella, sixteen in B. rapa and no TN encoding gene in E. salsugineum. Within the phylogenetic tree, five clades with orthologous proteins were identified (Additional file 4: Figure S2). None of the clades contained proteins from all four species.
A complete RLM3 sequence was present in 13 out of 19 A. thaliana accessions including Col-0 and no transcripts lacking one or more domains were identified. The high $\mathrm{Ka} /$ $K s$ ratio of 2.3 suggests that $R L M 3$ is under positive selection in the 13 accessions. Examination of the chromosome region spanning the RLM3 locus revealed that approximately $8,200 \mathrm{bp}$ in Col-0 was completely absent in six accessions (Kn-0, Rsch-4, Tsu-0, Wil-2, Ws-0 and Wu-0), while the flanking genes; At4g16980 and At4g17000 were present (Figure 3D). The At4g17000 gene has experienced mutations and small deletions, resulting in early stop codons. The approximately 400 bp between At4g16980 and At4g17000 not found in the Col-0 genomic sequence showed minor polymorphisms between these six accessions indicating that the deletion of $R L M 3$ resulted from a single event.

A RLM3-like gene was found in A. lyrata (clade no. 3; Additional file 4: Figure S2) suggesting the presence of RLM3 before the split from $A$. thaliana 13 Mya [36]. In contrast, no RLM3 homolog was found in the C. rubella, $B$. rapa and $E$. salsugineum genome sequences. To further trace a possible origin of $R L M 3$, the BRX domain was used in phylogenetic analysis but no orthology could be found to sequences within the kingdom Plantae (Additional file 10: Figure S5). We conclude that RLM3 has most likely evolved entirely within the genus of Arabidopsis.

\section{Discussion}

In this report we describe a genome-wide survey of the large $R$ gene family in $19 \mathrm{~A}$. thaliana accessions and four related species in the Brassicaceae family. The comparisons of the A. thaliana accessions revealed a great variation in gene numbers and a biased loss of LRR domains. Interestingly, the Col-0 genome was the most $R$ gene dense accession in the dataset. We checked for biases in the re-sequencing and gene annotation process of the additional $A$. thaliana genotypes but could not identify any obvious explanation for loss of $R$ genes in these accessions. This is in line with a recent genome study comprising de novo assembly of $180 \mathrm{~A}$. thaliana accessions, which revealed large variation in genome size, with 1.3-3.3 Mb of new sequences and 200-300 additional genes per genotype [37]. The differences were however found to be mainly due to $45 \mathrm{~S}$ rDNA copies and no new $R$ genes absent in Col-0 was reported.

Col- 0 is a direct descendent of Col-1 and was selected from a Landsberg population based on its fertility, and vigorous plant growth [16]. The same population was used in irradiation experiments, resulting in the Landsberg erecta accessions (Ler). It has now become clear that the original Landsberg population contained a mixture of slightly different genotypes, explaining the observed difference in $R$ gene repertoire between Col- 0 and Ler- 0 . The genetic variation among $A$. thaliana accessions as observed in our dataset 
has a long history of being exploited for $R$ gene mapping and cloning. Characterization of resistance genes to $P$. syringae (RPM, RPS) together with RPP genes to the oomoycete Hyaloperonospora arabidopsidis have been in the forefront and also advanced the understanding of interactions with pathogen effectors. The RPP1 locus of the Ws- 0 and $\mathrm{Nd}-1$ accessions recognize different $H$. arabidopsidis isolates, an observation that lead to the discovery of the avirulence gene ATR1 and six divergent alleles [38]. Sequence alignment with ATR1 syntenic genes in Phytophthora sojae and P. infestans in turn revealed the RxLR translocation core motif, adding another dimension to the genetic makeup of hostpathogen pairs and effector biology.

Within the 18 accessions of $A$. thaliana a large number of $R$ genes were missing one or more domains in comparison to Col-0, with the loss of LRR domains as the most common alteration. Modulation of the LRR sequences together with gene conversion, domain swapping and deletion events are suggested strategies for a plant to coevolve with a pathogen. LRR domains have been identified in a diverse variety of bacterial, protist and fungal species, together representing thousands of genes [12]. Fusion of the LRR domains with the NB domain is of a more recent origin than LRR fusion with receptor-like kinases, which are seen only in the land plant lineage. The LRR domain is suggested to have evolved several times resulting in eight specific classes, which differ in sequence length and similarity within the variable segment of the LRR domain $[39,40]$. One of the LRR classes, referred to as Plant Specific LRRs has been shown to be under diversifying selection in several $R$ proteins [41-44]. This type of sequence diversifications most likely reflects co-evolution with pathogen effectors, proteins known to directly or indirectly interact with the LRR motifs [7,45-47]. The importance of presence or absence of a particular LRR domain has also been demonstrated. In the absence of the P. syringae effector AvrPphB, the LRR domain of RPS5 inhibits the activity of the CC and NB domains [48]. Consequently, loss of the LRR suppressor activity results in plant cell death due to constitutive RPS5 activity. It was therefore not surprising that none of the RPS5 homologs in our dataset lacked the LRR domain. RPS2, RPS4 and RPS6 sequences were highly conserved between accessions and the LRR domains showed low degree of polymorphisms $(K a / K s$ ratio between 0.64 and 0.76$)$. In case of RPS4 the LRR domain is important for protein stability but it lacks the suppressor activity, like RPS5 [49].

In many $A$. thaliana accessions in our dataset we found $R$ genes encoding bipartite proteins, often represented by the loss of the LRR domain in comparison to Col-0. Such TN-encoding genes have been speculated to function as adapter proteins interacting with TNL proteins or with downstream signaling components [17]. For example, PBS1, an important player in the RPS5 defense response, was found to interact with a TN protein [50]. Whether $\mathrm{CN}$ and $\mathrm{TN}$ genes in general act in protein complexes recognizing pathogen effectors remains to be demonstrated. Plant $R$ genes encoding bipartite proteins also have been speculated to be part of an evolutionary reservoir in plants, allowing the formation of new genes through duplications, translocation and fusion $[12,51,52]$. The fusion between the TN and BRX domain in RLM3 is unique for $A$. thaliana and A. lyrata, possible dimerizing with other BRX domaincontaining proteins, since homo- and heterodimerization capability between BRX domains of individual proteins has been shown [53]. Further, the transcription factor BRX, containing two BRX domains was shown to control the expression of a gene important in brassinolide synthesis [54] and thereby modulate both plant root and shoot growth.

In our dataset we observed a great variation in the number of unique CNL and TNL $R$ genes, ranging from 33 in E. salsugineum to 63 in B. rapa. Copy number differences within different species of the $R$ gene family is proposed to be driven by gene loss through pseudogenization or expansion through duplication events and subsequent divergence [12]. The five species in our dataset represent two lineages; lineage I (Arabidopsis and Capsella) and lineage II (Brassica and Eutrema), diverging at approximately 43 Mya $[36,55]$. Due to the close relationship between the five species, higher numbers of conserved $R$ genes was expected, but no lineage-specific $R$ gene repertoires were found. Comparative genomic analysis between $A$. thaliana and B. rapa already established orthology between several NB-LRR genes [24]. However, in our study we found eleven additional sets including orthologs to ADR1-L1, ADR1-L2, RPP1, RPP13 and ZAR1. Out of the $528 R$ genes analyzed, only two CNLs and three TNLs were conserved in the five species. One of these, ZAR1, is also present in many other species within the eudicots, mainly within the Rosid clade [22]. The Rosid clade diverged from the Caryophyllales and Asterids more than 110 Mya [56] suggesting an ancient origin of the ZAR1 gene. Recently it was shown that ZAR1 interacts with the pseudokinase ZED1 in mediating immunity to $P$. syringae [57]. This pseudokinase family is also common among flowering plants and it could be speculated that pseudokinases and ZAR1 plays a general role in basal plant defense responses not seen in the ETI response triggered by $P$. syringae in $A$. thaliana.

\section{Conclusions}

Here, we have revealed a large variation in the $R$ gene repertoire in the $A$. thaliana accessions, highlighting both the fast evolving nature of the $R$ gene family but also a potential bias in the usage of a single genotype for genome comparisons. The recent advances in genome sequencing technologies enable re-sequencing of genotypes of interest for crop 
improvements with reasonable costs and rapid generation of molecular markers that co-segregate with traits of interest. An abundant supply of gene information from the rich genetic resources of Brassica species can therefore be foreseen along with methods for enrichment of genes of interests. Using such strategies, the number of NB-LRR genes in the potato genome was increased from 438 to 755 [58], demonstrating new avenues and breakthroughs made possible by next generation sequencing in the relatively short time that has passed since the sequencing of the first flowering plant.

\section{Methods}

\section{Data sampling}

The coding (CDS) and protein sequences of the $A$. thaliana Col-0 reference genome, 18 A. thaliana accessions, A. lyrata, C. rubella, B. rapa and E. salsugineum (previously Thellungiella halophila) genomes were downloaded from online databases [19,59-66]. Proteins with significant match according to the Pfam software [67] with the TIR domain (PF01582), NB-ARC (NB) domain (PF00931), and LRR domains (LRR1-5, 7-8), (PF00560, PF07723, PF07725, PF12799, PF13306, PF13504, PF13855) were selected. All proteins lacking the TIR domain were analyzed for the presence of the CC region with the COILS server using default settings and a confidence threshold $>0.9$ [68]. For the $A$. thaliana reference genome of Col-0 and the four species, genes encoding a TIR domain in combination of a NB and LRR (TNL) or a CC in combination with a NB and LRR (CNL) domains were selected. In the case of different isoforms, the longest transcript of each gene was included in the dataset. All protein sequences were subjected to Pfam homology and COILS server searches to identify CNL or TNL as described above for the $A$. thaliana accessions.

The RESISTANCE TO LEPTOSPHAERIA MACULANS 1 (RLM1) and RESISTANCE TO LEPTOSPHAERIA MACULANS 3 (RLM3) loci were selected for detailed analysis. Genomic and CDS sequences spanning two genes upstream (At1g63710) and downstream (At1g64090) of the RLM1 locus [26] were retrieved from the TAIR10 database [16]. The CDS sequences of At1g63710 through At1g64090 in Col-0 were used to identify the corresponding chromosomal regions in A. lyrata, C. rubella, B. rapa, and E. salsugineum by BLAST search against the Phytozome database [60,69]. Similarly, the At4g16980-At4g17000 region around the RLM3 locus (At4g16990) [34] was selected and identified in A. lyrata, C. rubella, B. rapa, and E. salsugineum. The Pfam software was used to select genes encoding a combination of TIR and NB domains (TN) in Col-0 and subsequent orthologs in the $18 \mathrm{~A}$. thaliana accessions were identified. For the presence/absence (P/A) polymorphisms of the NB-LRR genes the definition of [70] was used. The average non-synonymous and synonymous substitutions per site ratio $(K a / K s)$ for each gene were determined using the number of differences with the Nei-Gojobori distance method implemented in MEGA 5.2 [71].

\section{Multiple sequence alignment and phylogenetic analysis}

The NB domains in the CNL and TNL proteins identified in A. lyrata, C. rubella, B. rapa and E. salsugineum genomes were aligned with ClustalW [72] using default settings and the alignment translated to nucleotides with the TranslatorX tool [73]. Poorly aligned sites were removed from the dataset using GBlocks 0.91b [74] with following settings: $-\mathrm{b} 1=282,-\mathrm{b} 2=283,-\mathrm{b} 4=5,-\mathrm{b} 5=\mathrm{h},-\mathrm{b} 6=\mathrm{y}$. Identical proteins were reduced to one representative. A neighbor-joining tree was constructed using PAUP* $4.0 \beta 10$ [75] through Geneious version 7.0.4 [76] using the GTR+G+I model with a 0.1 proportion of invariable sites and 1,000 bootstrap replicates. Proteins with a bootstrap confidence $\geq 70$ were selected as orthologous. To further analyze parts of the resulting tree, a maximum likelihood $(\mathrm{ML})$ analysis was performed using the GTR+G+I model and 1,000 bootstrap rates replicates in MEGA 5.2 [71]. Proteins with a BREVIS RADIX (BRX) domain were identified in BLASTP homology searches using a hidden Markov model (HMM) of the BRX domain sequence (PF08381). The BRX domain sequences were aligned and translated to nucleotides with translatorX and a ML tree was constructed in MEGA 5.2 using the $\mathrm{GTR}+\mathrm{G}+\mathrm{I}$ rates and 1,000 bootstrap replicates.

\section{Analysis of the RLM1 and RLM3 loci}

Syntenic orthologs between $A$. thaliana Col-0, A. lyrata, C. rubella, B. rapa, and E. salsugineum were identified using the SynOrths v1.0 tool with default settings [77], by comparing all genes in the selected region between all pairs of species. Protein pairs with an $E$-value cutoff of $<1 \mathrm{e}-9$ were considered orthologous. All none-TNL proteins within the $R L M 1$ region in the different species were assigned to orthologous groups using the OrthoMCL version 2.0 server [78] followed by Pfam homology search to identify domain architecture. TNL proteins and the unannotated regions within the RLM1 locus in the different species were aligned using ClustalW, manually inspected and classified as highly similar ( $\geq 60 \%$ aa identity) or orthologous ( $\geq 80$ aa identity). The evolutionary $p$-distance (the proportion of amino acid sites at which two sequences are different divided by the total number of sites converted to percentages) between the TNL genes in the RLM1 region of the $18 \mathrm{~A}$. thaliana accessions [19] was calculated in comparison to Col-0 [79]. For the RLM3 locus, the region between At4g16980-At4g17000 in A. thaliana Col-0, A. lyrata, C. rubella, B. rapa and E. salsugineum were aligned using ClustalW with the default settings and manually inspected.

To PCR amplify the $R L M 1 A$ region in different species, DNA was extracted by dissolving crushed leaves of $A$. 
lyrata, (I2_AUT1 [80]), C. rubella (Cr1GR1, Samos, Greece), B. rapa ssp. pekinensis cv. 'Granaat', B. napus Surpass 400 and $B$. rapa ssp. sylvestris in extraction buffer (50 mM Tris, pH 7.9; 0.06 mM EDTA, pH 8; $0.62 \mathrm{mM}$ Triton X-100 and $50 \mathrm{mM} \mathrm{LiCl}$ ) followed by incubation at $55^{\circ} \mathrm{C}$ for $10 \mathrm{~min}$. DNA was purified by phenol/chloroform/isoamyl alcohol (25:24:1) followed by chloroform/ isoamyl alcohol (24:1), and precipitated with $3 \mathrm{M} \mathrm{NaOAc}$ (pH 5.2) and 100\% ethanol. The RLM1A region containing part of the flanking genes (AT1G64065 and AT1G64080 in A. thaliana) was PCR amplified in C. rubella $(\mathrm{Cr})$, A. lyrata $(\mathrm{Al})$ and $B$. rapa ssp. pekinensis $(\mathrm{Br})$ using species specific primers, Cr_Fw: GTTGTGGTTGAGATCGGTTC, Cr_Rv: TGTTGCACGAAAAGAGACAA, Al_Fw: GAACCTCCA GGGAAATGTCT, Al_Rv: CCATTGTCACTTCCGTTAC C, Br_Fw: CACTTCCCCCATTAACTCCT and Br_Rv: TAAAAGCGGAGAGGGAGATT. In Surpass 400 and $B$. rapa ssp. sylvestris $R L M 1 A$ was amplified using $\mathrm{RL}$ M1A_Fw3: CATCCCATTGGTCTTGATGA and RLM A_Rv3: TGGCTTTCACAAGATCACCA. The PCR products were purified using the GeneJET PCR purification kit (Thermo Scientific) followed by sequencing (Macrogen Inc. Amsterdam, the Netherlands).

\section{Availability of supporting data}

The data supporting the results of this article are included within the article.

\section{Additional files}

Additional file 1: Table S1. List of $R$ genes in the genomes of $A$. thaliana, A. lyrata, C. rubella, B. rapa and E. salsugineum. Nomenclature is according to Phytozome or otherwise stated. Identifiers in B. rapa are according to [81]. *Plant Resistance Gene Wiki [82], **Uniprot [83], §Not used in the Neighbor Joining analysis.

Additional file 2: Table S2. List of $R$ genes in A. thaliana with known function used in this study $[22,26,28,34,41,84-100]$.

Additional file 3: Figure S1. Chromosomal distribution of conserved and selected NB-LRR genes in 19 A. thaliana accessions. On the right side of each chromosome the 29 conserved CNL and TNL genes are depicted together with orthologs in A. Iyrata, C. rubella, B. rapa, and E. salsugineum in blue. The red genes have orthologs in the four Brassicaceae species but are absent in several of the $A$. thaliana accessions. Genes on the left side of the chromosomes are attributed to a defense response but were not found conserved between the 19 accessions. $R$ gene information is compiled in Additional file 2: Table S2.

Additional file 4: Figure S2. Phylogenetic analysis based on the NB domain in R proteins from A. thaliana, A. lyrata, C. rubella, B. rapa, and E. salsugineum. The neighbor joining tree was constructed using the GTR model and 1,000 bootstrap replicates. Orthologous proteins were identified in individual clades at a bootstrap value of $\geq 70$ and are highlighted and numbered. Labeling is as follows: CNL proteins (green), TNL proteins (blue), TN proteins (light blue) and clades with bootstrap $<70$ (grey). The identifiers of each gene are described in Additional file 1: Table S1.

Additional file 5: Table S3. Orthologous $R$ genes between $A$. thaliana A. lyrata, C. rubella, B. rapa and E. salsugineum.

Additional file 6: Figure S3. Maximum likelihood analysis of ten CNL genes. The construction of the maximum likelihood tree was done using the alignment of the complete CDS sequence of the ten sequences in clade 12 (CNL) in Additional file 4: Figure S2. The GTR model was used and bootstrapping was with 1,000 replicates. The identifiers of each gene are described in Additional file 1: Table S1.

Additional file 7: Figure S4. Synteny in the RLM1 locus between five species. In (A) between A. Iyrata (A), A. thaliana (Col-0) (Al) and C. rubella $(\mathrm{Cr})$ and $(\mathrm{B})$ between B. rapa $(\mathrm{Cr})$, A. thaliana (Col-0) and E. salsugineum (Es). The seven RLM1 genes; RLM1A (A, At1g64070), RLM1B, (B, At1g63880), RLM1C, (C, At1g63870), RLM1D, (D, At1g63860), RLM1E (E, At1g63750), RLM1F (F, At1g63740) and RLM1G (G, At1g63730) and the other TNL encoding genes in the four species are in orange (light orange if un-annotated). The non-TNL genes are depicted in black (synteny) or white (no synteny). Synteny between genes is depicted dotted lines showing similarity between two TNL proteins with an identity of 60 or higher. Reduction in bp length is depicted by the double forward slashes.

Additional file 8: Table S4. Distribution of presence and absence of gene members in the RLM1 locus in 19 A. thaliana accessions.

Additional file 9: Table S5. $p$-distance of the different TNL encoding genes in the RLM1 locus in the 19 A. thaliana accessions.

Additional file 10: Figure S5. Maximum likelihood analysis of the BRX domain. The GTR model was used and bootstrapping was with 1,000 replicates. Labeling is as follows: dicots (green), monocots (dark blue), green algae (orange), moss (pink) and lycophyta (light blue). The clades consisting of BRX domains of RLM3 is highlighted.

\section{Competing interests}

The authors declare that they have no competing interests.

\section{Authors' contributions}

HMP, NG and CD conceived and designed the study; HMP, NG and JF compiled and analyzed the data; HMP and CD wrote the manuscript. All authors read and approved the final manuscript.

\section{Acknowledgements}

The authors would like to thank Joel Sohlberg for guidance on the phylogenetic analyses. This work was supported by the following foundations: Nilsson-Ehle, Helge Ax:son Johnson; and the Research Councils VR and Formas together with the Swedish University of Agricultural Sciences.

Received: 13 June 2014 Accepted: 20 October 2014

Published online: 01 November 2014

\section{References}

1. Chisholm ST, Coaker G, Day B, Staskawicz BJ: Host-microbe interactions: shaping the evolution of the plant immune response. Cell 2006, 124:803-814.

2. Jones JDG, Dangl JL: The plant immune system. Nature 2006, 444:323-329.

3. Zipfel C: Pattern-recognition receptors in plant innate immunity. Curr Opin Immunol 2008, 20:10-16.

4. Macho AP, Zipfel C: Plant PRRs and the activation of innate immune signaling. Mol Cell 2014, 54:263-272.

5. Mackey D, Holt BF, Wiig A, Dangl JL: RIN4 interacts with Pseudomonas syringae type III effector molecules and is required for RPM1-mediated resistance in Arabidopsis. Cell 2002, 108:743-754.

6. Kim MG, da Cunha L, McFall AJ, Belkhadir Y, DebRoy S, Dangl JL, Mackey D: Two Pseudomonas syringae type III effectors inhibit RIN4-regulated basal defense in Arabidopsis. Cell 2005, 21:749-759.

7. Jia Y, McAdams SA, Bryan GT, Hershey HP, Valent B: Direct interaction of resistance gene and avirulence gene products confers rice blast resistance. EMBO J 2000, 19:4004-4014.

8. Kanzaki H, Yoshida K, Saitoh H, Fuijsaki K, Hirabuchi A, Alaux L, Fournier E, Tharreau D, Terauchi R: Arms race co-evolution of Magnaporthe oryzae AVR-Pik and rice Pik genes driven by their physical interactions. Plant J 2012, 72:894-907.

9. DeYoung BJ, Innes RW: Plant NBS-LRR proteins in pathogen sensing and host defense. Nat Immunol 2006, 7:1243-1249.

10. Dodds PN, Rathjen JP: Plant immunity: towards an integrated view of plant-pathogen interactions. Nat Rev Genet 2010, 11:539-548. 
11. Maekawa T, Kufer TA, Schulze-Lefert P: NLR functions in plant and animal immune systems: so far and yet so close. Nat Immunol 2011, 12:818-826.

12. Yue JX, Meyers BC, Chen JQ, Tian D, Yang S: Tracing the origin and evolutionary history of plant nucleotide-binding site-leucine-rich repeat (NBS-LRR) genes. New Phytol 2012, 193:1049-1063

13. Hammond-Kosack KE, Parker JE: Deciphering plant-pathogen communication: fresh perspectives for molecular resistance breeding. Curr Opin Biotechnol 2003, 14:177-193.

14. Fuller VL, Lilley CJ, Urwin PE: Nematode resistance. New Phytol 2008, 180:27-44.

15. Vleeshouwers VGAA, Raffaele S, Vossen JH, Champouret N, Oliva R, Segretin ME, Rietman H, Cano LM, Lokossou A, Kessel G, Pel MA, Kamoun S: Understanding and exploiting late blight resistance in the age of effectors. Annu Rev Phytopathol 2011, 49:507-531.

16. TAIR10 [ftp://ftp.arabidopsis.org/home/tair/]

17. Meyers BC, Morgante M, Michelmore RW: TIR-X and TIR-NBS proteins: two new families related to disease resistance TIR-NBS-LRR proteins encoded in Arabidopsis and other plant genomes. Plant J 2002, 32:77-92.

18. Meyers BC, Kozik A, Griego A, Kuang H, Michelmore RW: Genome-wide analysis of NBS-LRR-encoding genes in Arabidopsis. Plant Cell 2003, 15:809-834.

19. Gan X, Stegle O, Behr J, Steffen JG, Drewe P, Hildebrand KL, Lyngsoe R, Schultheiss SJ, Osborne EJ, Sreedharan VT, Kahles A, Bohnert R, Jean G, Derwent P, Kersey P, Belfield EJ, Harberd NP, Kemen E, Toomajian C, Kover PX, Clark RM, Rätsch G, Mott R: Multiple reference genomes and transcriptomes for Arabidopsis thaliana. Nature 2011, 477:419-423.

20. Chen Q, Han Z, Jiang H, Tian D, Yang S: Strong positive selection drives rapid diversification of $R$-genes in Arabidopsis relatives. J Mol Evol 2010, 70:137-148.

21. Guo YL, Fitz J, Schneeberger K, Ossowski S, Cao S, Weigel D: Genome-wide comparison of nucleotide-binding site leucine-rich repeat-encoding genes in Arabidopsis. Plant Physiol 2011, 157:757-769.

22. Lewis JD, Wu R, Guttman DS, Desveaux D: Allele-specific virulence attenuation of the Pseudomonas syringae HopZ1a type III effector via the Arabidopsis ZAR1 resistance protein. PLoS Genet 2010, 6:e1000894.

23. Wroblewski T, Coulibaly S, Sadowski J, Quiros CF: Variation and phylogenetic utility of the Arabidopsis thaliana Rps 2 homolog in various species of the tribe Brassiceae. Mol Phylogenet Evol 2000, 16:440-448.

24. Yu J, Therim S, Zhang F, Tong C, Huang J, Cheng X, Dong C, Zhou Y, Qin R, Hua W, Liu S: Genome-wide comparative analysis of NBS-encoding genes between Brassica species and Arabidopsis thaliana. BMC Genomics 2014, 15:3.

25. Fitt BDL, Brun H, Barbetti MJ, Rimmer SR: World-wide importance of phoma stem canker (Leptosphaeria maculans and L. biglobosa) on oilseed rape (Brassica napus). Eur J Plant Pathol 2006, 114:3-15.

26. Staal J, Kaliff M, Bohman S, Dixelius C: Transgressive segregation reveals two Arabidopsis TIR-NB-LRR resistance genes effective against Leptosphaeria maculans, causal agent of blackleg disease. Plant J 2006, 46:218-230.

27. Hall SA, Allen RL, Baumber RE, Baxter LA, Fisher K, Bittner-Eddy PD, Rose LE, Holub EB, Beynon JL: Maintenance of genetic variation in plants and pathogens involves complex networks of gene-for-gene interactions. Mol Plant Pathol 2009, 10:449-457.

28. Goritschnig S, Krasileva KV, Dahlbeck D, Staskawicz BJ: Computational prediction and molecular characterization of an oomycete effector and the cognate Arabidopsis resistance gene. PLoS Genet 2012, 8:e1002502.

29. Bohman S, Staal J, Thomma BPHJ, Wang M, Dixelius C: Characterisation of an Arabidopsis-Leptosphaeria maculans pathosystem: resistance partially requires camalexin biosynthesis and is independent of salicylic acid, ethylene and jasmonic acid signaling. Plant J 2004, 37:9-20.

30. Gos G, Slotte T, Wright SI: Signatures of balancing selection are maintained at disease resistance loci following mating system evolution and a population bottleneck in the genus Capsella. BMC Evol Biol 2012, 12:152.

31. Warwick SI: Brassicaceae in Agriculture. In Genetics and Genomics of the Brassicaceae. Volume 9. Edited by Schmidt R, Bancroft I. New York: Springer Verlag; 2011:33-66.

32. Mithen RF, Lewis BG, Heaney RK, Fenwick GR: Resistance of leaves of Brassica species to Leptosphaeria maculans. Trans Brit Mycol Soc 1987, 88:525-531.

33. Crouch JH, Lewis BG, Mithen RF: The effect of A genome substitution on the resistance of Brassica napus to infection by Leptosphaeria maculans. Plant Breed 1994, 112:265-278.

34. Staal J, Kaliff M, Dewaele E, Persson M, Dixelius C: RLM3, a TIR domain encoding gene involved in broad-range immunity of Arabidopsis to necrotrophic fungal pathogens. Plant J 2008, 55:188-200.
35. Mouchel CF, Briggs GC, Hardtke CS: Natural genetic variation in Arabidopsis identifies BREVIS RADIX, a novel regulator of cell proliferation and elongation in the root. Genes Dev 2004, 18:700-714.

36. Beilstein MA, Nagalingum NS, Clements MD, Manchester SR, Mathews S: Dated molecular phylogenies indicate a Miocene origin for Arabidopsis thaliana. Proc Natl Acad Sci U S A 2010, 107:18724-18728.

37. Long Q, Rabanal FA, Meng D, Huber CD, Farlow A, Platzer A, Zhang Q, Vilhjálmsson BJ, Korte A, Nizhynska V, Voronin V, Korte P, Sedman L, Mandáková T, Lysak MA, Seren Ü, Hellmann I, Nordborg M: Massive genomic variation and strong selection in Arabidopsis thaliana lines from Sweden. Nat Genet 2013, 45:884-891.

38. Rehmany AP, Gordon A, Rose LE, Allen RL, Armstrong MR, Whisson SC, Kamoun S, Tyler BM, Birch PRJ, Beynon JL: Differential recognition of highly divergent downy mildew avirulence gene alleles by RPP1 resistance genes from two Arabidopsis lines. Plant Cell 2005, 17:1839-1850

39. Kajava AV, Anisimova M, Peeters N: Origin and evolution of GALA-LRR, a new member of the CC-LRR subfamily: from plants to bacteria? PLOS ONE 2008, 3:e1694.

40. Miyashita $\mathrm{H}$, Kuroki $Y$, Kretsinger RH, Matsushima N: Horizontal gene transfer of plant-specific leucine-rich repeats between plants and bacteria. Nature 2013, 5:580-598.

41. McDowell JM, Dhandaydham M, Long TA, Aarts MGM, Goff S, Holub EB, Dangl $J$ : Intragenic recombination and diversifying selection contribute to the evolution of downy mildew resistance at the RPP8 locus of Arabidopsis. Plant Cell 1998, 10:1861-1874.

42. Meyers BC, Shen KA, Rohani P, Gaut BS, Michelmore RW: Receptor-like genes in the major resistance locus of lettuce are subject to divergent selection. Plant Cell 1998, 11:1833-1846.

43. Ellis J, Dodds P, Pryor T: Structure, function and evolution of plant disease resistance genes. Curr Opin Plant Biol 2000, 3:278-284.

44. Ng A, Xavier RJ: Leucine-rich repeat (LRR) proteins: integrators of pattern recognition and signaling in immunity. Autophagy 2011, 7:1082-1084.

45. Deslandes L, Olivier J, Theulières F, Hirsch J, Feng DX, Bittner-Eddy P, Beynon J, Marco Y: Resistance to Ralstonia solanacearum in Arabidopsis thaliana is conferred by the recessive RRS1-R gene, a member of a novel family of resistance genes. Proc Natl Acad Sci U S A 2002, 99:2404-2409.

46. Axtell MJ, Staskawicz BJ: Initiation of RPS2-specified disease resistance in Arabidopsis is coupled to the AvrRpt2-directed elimination of RIN4. Cell 2003, 112:369-377.

47. Mackey D, Belkhadir Y, Alonso JM, Ecker JR, Dangl JL: Arabidopsis RIN4 is a target of the type III virulence effector AvrRpt2 and modulates RPS2mediated resistance. Cell 2003, 112:379-389.

48. Qi D, DeYoung BJ, Innes RW: Structure-function analysis of the coiled-coil and leucine-rich repeat domains of the RPS5 disease resistance protein. Plant Physiol 2012, 158:1819-1832.

49. Zhang Y, Dorey S, Swiderski M, Jones JDG: Expression of RPS4 in tobacco induces an AvrRps4-independent HR that requires EDS1, SGT and HSP90. Plant J 2004, 40:213-224.

50. Nandety RS, Caplan JL, Cavanaugh K, Perroud B, Wroblewski T, Michelmore RW, Meyers BC: The role of TIR-NBS and TIR-X proteins in plant basal defense responses. Plant Physiol 2013, 162:1459-1472.

51. Jacob F, Vernaldi S, Maekawa T: Evolution and conservation of plant NLR functions. Front Immunol 2013, 4:297

52. Joshi RK, Nayak S: Perspectives of genomic diversification and molecular recombination towards $R$-gene evolution in plants. Physiol Mol Biol Plants 2013, 19:1-9.

53. Briggs GC, Mouchel CF, Hardtke CS: Characterization of the plant-specific BREVIS RADIX gene family reveals limited genetic redundancy despite high sequence conservation. Plant Physiol 2006, 140:1306-1316.

54. Mouchel CF, Osmont KS, Hardtke CS: BRX mediates feedback between brassinosteroid levels and auxin signalling in root growth. Nature 2006, 443:458-461.

55. Couvreur TLP, Franzke A, Al-Shehbaz IA, Bakker FT, Koch MA, Mummenhoff K Molecular phylogenetics, temporal diversification, and principles of evolution in the mustard family (Brassicaceae). Mol Biol Evol 2010, 27:55-71.

56. Magallón S, Hilu KW, Quandt D: Land plant evolutionary timeline: gene effects are secondary to fossil constraints in relaxed clock estimation of age and substitution rates. Am J Bot 2013, 100:556-573.

57. Lewis JD, Lee AHY, Hassan JA, Wan J, Hurley B, Jhingree JR, Wang PW, Lo T, Youn JY, Guttman DS, Desveaux D: The Arabidopsis ZED1 pseudokinase is required for 
ZAR1-mediated immunity induced by the Pseudomonas syringae type III effector HopZ1a. Proc Natl Acad Sci U S A 2013, 110:18722-18727.

58. Jupe F, Witek K, Verweij W, Śliwka J, Pritchard L, Etherington GJ, Maclean D, Cock PJ, Leggett RM, Bryan GJ, Cardle L, Hein I, Jones JD: Resistance gene enrichment sequencing (RenSeq) enables reannotation of the NB-LRR gene family from sequenced plant genomes and rapid mapping of resistance loci in segregating populations. Plant J 2013, 76:530-544.

59. 19 genomes of Arabidopsis thaliana [http://mus.well.ox.ac.uk/19genomes/]

60. Phytozome [http://www.phytozome.net/]

61. Hu TT, Pattyn P, Bakker EG, Cao J, Cheng JF, Clark RM, Fahlgren N, Fawcett JA, Grimwood J, Gundlach H, Haberer G, Hollister JD, Ossowski S, Ottilar RP, Salamov AA, Schneeberger K, Spannagl M, Wang X, Yang L, Nasrallah ME, Bergelson J, Carrington JC, Gaut BS, Schmutz J, Mayer KFX, Van de Peer Y, Grigoriev IV, Nordborg M, Weigel D, Guo YL: The Arabidopsis lyrata genome sequence and the basis of rapid genome size change. Nat Genet 2011, 43:476-483.

62. JGl genome portal [http://genome.jgi-psf.org/Araly1/Araly1.download.ftp.html]

63. Slotte T, Hazzouri KM, Ågren JA, Koenig D, Maumus F, Guo YL, Steige K, Platts AE, Escobar JS, Newman LK, Wang W, Mandáková T, Vello E, Smith LM, Henz SR, Steffen J, Takuno S, Brandvain Y, Coop G, Andolfatto P, Hu TT, Blanchette M, Clark RM, Quesneville H, Nordborg M, Gaut BS, Lysak MA, Jenkins J, Grimwood J, Chapman J, et al: The Capsella rubella genome and the genomic consequences of rapid mating system evolution. Nat Genet 2013, 45:831-837

64. Wang $X$, Wang $H$, Wang J, Sun R, Wu J, Liu S, Bai Y, Mun JH, Bancroft I, Cheng F, Huang S, Li X, Hua W, Wang J, Wang X, Freeling M, Pires JC, Paterson AH, Chalhoub B, Wang B, Hayward A, Sharpe AG, Park BS, Weisshaar B, Liu B, Li B, Liu B, Tong C, Song C, Duran C, et al: The genome of the mesopolyploid crop species Brassica rapa. Nat Genet 2011, 43:1035-1040.

65. Yang R, Jarvis DE, Chen H, Beilstein MA, Grimwood J, Jenkins J, Shu S, Prochnik S, Xin M, Ma C, Schmutz J, Wing RA, Mitchell-Olds T, Schumaker KS, Wang $X$ : The reference genome of the halophytic plant Eutrema salsugineum. Front Plant Sci 2013, 4:46.

66. Sanseverino W, Hermoso A, D'Alessandro R, Vlasova A, Andolfo G, Frusciante L, Lowy E, Roma G, Ercolano MR: PRGdb 2.0: towards a community-based database model for the analysis of R-genes in plants. Nucleic Acids Res 2013, 41:D1167-D1171.

67. Punta M, Coggill PC, Eberhardt RY, Mistry J, Tate J, Boursnell C, Pang N, Forslund K, Ceric G, Clements J, Heger A, Holm L, Sonnhammer ELL, Eddy SR, Bateman A, Finn RD: The Pfam protein families database. Nucleic Acids Res 2012, 40:D290-D301.

68. Lupas A, Van Dyke M, Stock J: Predicting coiled coils from protein sequences. Science 1991, 252:1162-1164.

69. Goodstein DM, Shu S, Howson R, Neupane R, Hayes RD, Fazo J, Mitros T, Dirks W, Hellsten U, Putnam N, Rokhsar DS: Phytozome: a comparative platform for green plant genomics. Nucleic Acids Res 2011, 40:D1178-D1186

70. Tan S, Zhong Y, Hou H, Yang S, Tian D: Variation of presence/absence genes among Arabidopsis populations. BMC Evol Biol 2012, 12:86.

71. Tamura K, Peterson D, Peterson N, Stecher G, Nei M, Kumar S: MEGA5: molecular evolutionary genetics analysis using maximum likelihood, evolutionary distance, and maximum parsimony methods. Mol Biol Evol 2011, 28:2731-2739.

72. ClustalW [http://www.genome.jp/tools/clustalw/]

73. Abascal F, Zardoya R, Telford MJ: TranslatorX: multiple alignment of nucleotide sequences guided by amino acid translations. Nucleic Acids Res 2010, 38:W7-W13.

74. Castresana J: Selection of conserved blocks from multiple alignments for their use in phylogenetic analysis. Mol Biol Evol 2000, 17:540-552.

75. Swofford DL: PAUP* V4.068: Phylogenetic Analysis Using Parsimony * (and other Methods). Sunderland: Sinauer associates INC; 2002.

76. Biomatters, US [http://www.biomatters.com/\#/]

77. Cheng F, Wu J, Fang L, Wang X: Syntenic gene analysis between Brassica rapa and other Brassicaceae species. Front Plant Sci 2012, 3:198.

78. Fischer S, Brunk BP, Chen F, Gao X, Harb OS, lodice JB, Shanmugam D, Roos DS, Stoeckert CJ: Using OrthoMCL to assign proteins to OrthoMCL-DB groups or to Cluster proteomes into new ortholog groups. Curr Protoc Bioinformatics 2011, 35:6.12.1-6.12.19.

79. Nei M, Kumar S: Molecular Evolution and Phylogenetics. New York: Oxford University Press; 2000.

80. Jørgensen MH, Ehrich D, Schmickl R, Kock MA, Brysting AK: Interspecific and interploidal gene flow in Central European Arabidopsis (Brassicaceae). BMC Evol Biol 2011, 11:346.
81. Mun JH, Yu HJ, Park S, Park BS: Genome-wide identification of NBS-encoding resistance genes in Brassica rapa. Mol Genet Genomics 2009, 282:617-631.

82. Plant Resistance Gene Wiki [http://prgdb.crg.eu/wiki/Main_Page]

83. Uniprot [http://www.uniprot.org/]

84. Grant JJ, Chini A, Basu D, Loake GJ: Targeted activation tagging of the Arabidopsis NBS-LRR gene, ADR1, conveys resistance to virulent pathogens. Mol Plant Microbe Interact 2003, 8:669-680.

85. Bonardi V, Tang S, Stallmann A, Roberts M, Cherkis K, Dangl JL: Expanded functions for a family of plant intracellular immune receptors beyond specific recognition of pathogen effectors. Proc Natl Acad Sci U S A 2011, 108:16463-16468.

86. Borhan MH, Gunn N, Cooper A, Gulden S, Tör M, Rimmer SR, Holub EB: WRR4 encodes a TIR-NB-LRR protein that confers broad-spectrum white rust resistance in Arabidopsis thaliana to four physiological races of Albugo candida. Mol Plant Microbe Interact 2008, 21:757-768.

87. Faigón-Soverna A, Harmon FG, Storani L, Karayekov E, Staneloni RJ, Gassmann W, Más P, Casal JJ, Kay SA, Yanovsky MJ: A constitutive shadeavoidance mutant implicates TIR-NBS-LRR proteins in Arabidopsis photomorphogenic development. Plant Cell 2006, 18:2919-2928.

88. Lorang JM, Sweat TA, Wolpert TJ: Plant disease susceptibility conferred by a "resistance" gene. Proc Natl Acad Sci U S A 2007, 104:14861-14866.

89. Botella MA, Parker JE, Frost LN, Bittner-Eddy PD, Beynon JL, Daniels MJ, Holub EB, Jones JDG: Three genes of the Arabidopsis RPP1 complex resistance locus recognize distinct Peronospora parasitica avirulence determinants. Plant Cell 1998, 10:1847-1860

90. van der Biezen EA, Freddie CT, Kahn K, Parker JE, Jones JD: Arabidopsis RPP4 is a member of the RPP5 multigene family of TIR-NB-LRR genes and confers downy mildew resistance through multiple signalling components. Plant J 2002, 29:439-451.

91. Parker JE, Szabò V, Staskawicz BJ, Lister C, Dean C, Daniels MJ, Jones JDG: Phenotypic characterization and molecular mapping of the Arabidopsis thaliana locus RPP5, determining disease resistance to Peronospora parasitica. Plant J 1993, 4:821-831.

92. Bittner-Eddy P, Can C, Gunn N, Pinel M, Tör M, Crute I, Holub EB, Beynon J: Genetic and physical mapping of the RPP13 locus, in Arabidopsis, responsible for specific recognition of several Peronospora parasitica (downy mildew) isolates. Mol Plant Microbe Interact 1999, 12:792-802

93. Mindrinos M, Katagiri F, Yu GL, Ausubel FM: The A. thaliana disease resistance gene RPS2 encodes a protein containing a nucleotide-binding site and leucine-rich repeats. Cell 1994, 23:1089-1099.

94. Gassmann W, Hinsch ME, Staskawicz BJ: The Arabidopsis RPS4 bacterialresistance gene is a member of the TIR-NBS-LRR family of diseaseresistance genes. Plant J 1999, 20:265-277.

95. Warren RF, Henk A, Mowery P, Holub E, Innes RW: A mutation within the leucine-rich repeat domain of the Arabidopsis disease resistance gene RPS5 partially suppresses multiple bacterial and downy mildew resistance genes. Plant Cell 1998, 10:1439-1452.

96. Kim SH, Kwon SI, Saha D, Anyanwu NC, Gassmann W: Resistance to the Pseudomonas syringae effector HopA1 is governed by the TIR-NBS-LRR protein RPS6 and is enhanced by mutations in SRFR1. Plant Physio/ 2009, 150:1723-1732.

97. Stokes TL, Kunkel BN, Richards EJ: Epigenetic variation in Arabidopsis disease resistance. Genes Dev 2002, 16:171-182.

98. Zhang Z, Wu Y, Gao M, Zhang J, Kong Q, Liu Y, Ba H, Zhou J, Zhang Y: Disruption of PAMP-induced MAP kinase cascade by a Pseudomonas syringae effector activates plant immunity mediated by the NB-LRR protein SUMM2. Cell Host Microbe 2012, 11:253-263.

99. Eitas TK, Nimchuk ZL, Dangl JL: Arabidopsis TAO1 is a TIR-NB-LRR protein that contributes to disease resistance induced by the Pseudomonas syringae effector AvrB. Proc Natl Acad Sci U S A 2008, 105:6475-6480.

100. Nam M, Koh S, Kim SU, Domier LL, Jeon JH, Kim HG, Lee SH, Bent AF, Moon JS: Arabidopsis TTR1 causes LRR-dependent lethal systemic necrosis, rather than systemic acquired resistance, to tobacco ringspot virus. Mol Cells 2011, 32:421-429.

doi:10.1186/s12870-014-0298-z

Cite this article as: Peele et al:: Loss and retention of resistance genes in five species of the Brassicaceae family. BMC Plant Biology 2014 14:298. 